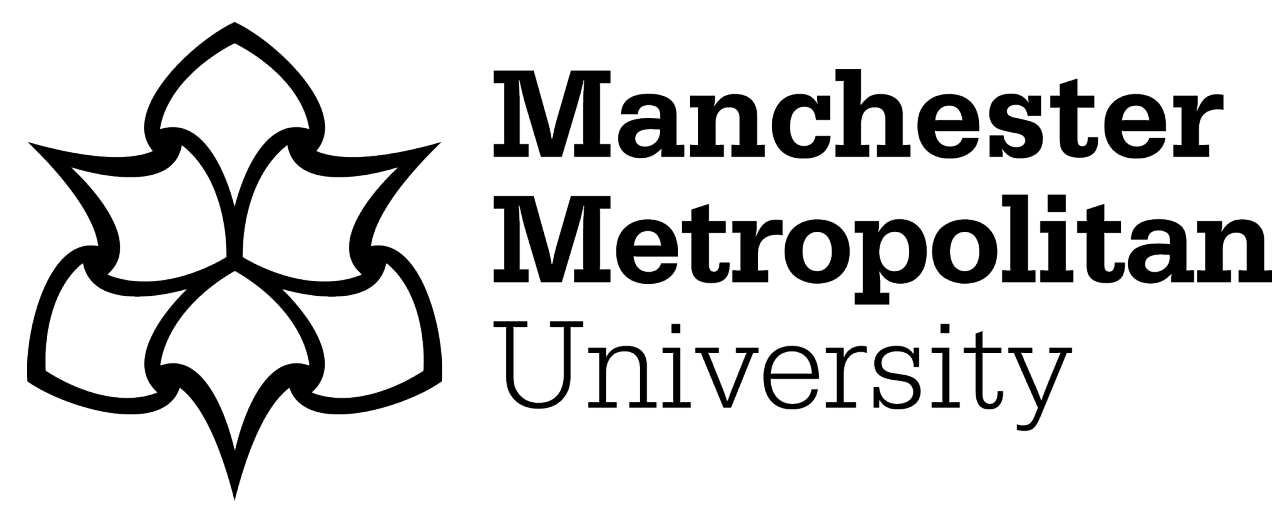

Persson, S, Benn, Yael, Dhingra, K, Owen, A, Clark-Carter, D and Grogan, Sarah (2018) Appearance-based interventions to reduce UV exposure: A systematic review. British Journal of Health Psychology, 23 (2). pp. 334-351. ISSN 1359-107X

Downloaded from: https://e-space.mmu.ac.uk/619741/

Version: Accepted Version

Publisher: Wiley

DOI: https://doi.org/10.1111/bjhp.12291

Please cite the published version 
APPEARANCE INTERVENTIONS TO REDUCE UV EXPOSURE

\title{
Appearance-based interventions to reduce UV exposure: A systematic review
}

\begin{abstract}
Purpose: As a majority of skin cancer cases are behaviourally preventable, it is crucial to develop effective strategies to reduce UV exposure. Health-focused interventions have not proved to be sufficiently effective, and it has been suggested that people might be more susceptible to information about the negative effects of the sun on their appearance.
\end{abstract}

Method: This systematic review of 30 separate papers, reporting 33 individual studies published between 2005 and 2017 assesses the overall effectiveness of appearance interventions on participants' UV exposure and sun protection behaviour.

Results: Appearance-based interventions have positive effects on sun exposure and sun protection, immediately after the intervention as well as up to 12 months afterwards. The meta-analysis found a medium effect size on sun protection intentions for interventions which combined UV photography and photoaging information: $\mathrm{r}+=.424 ; \mathrm{k}=3, \mathrm{~N}=319, \mathrm{CI}$ $=.279-.568, \mathrm{p}=.023$.

Conclusions: We provide a review of current research on the effectiveness of appearancebased interventions to reduce UV exposure. As well as highlighting methodological issues we recommend that practitioners administer a UV photo intervention in combination with photoaging information to reduce UV exposure. Furthermore, the review specifically recommends that future research focuses on the use of theoretical constructs to enhance photoaging information, and is conducted with older participants and in countries where people have less opportunity for sun exposure.

Keywords: Skin Cancer; UV Exposure; Appearance-based interventions; UV photography; Photoaging information 
APPEARANCE INTERVENTIONS TO REDUCE UV EXPOSURE

\section{Appearance-based interventions to reduce UV exposure: A systematic review}

Deaths from skin cancer are an increasing problem around the world; the World Health Organization (2015) reports that up to 2 million new cases occur globally each year. In the UK, non-melanoma skin cancers are by far the most common type of cancer with around 102, 000 new cases diagnosed annually (Cancer Research UK, 2016). There is an established link between ultraviolet (UV) radiation exposure and all types of skin cancer; this includes intentional (e.g. indoor and outdoor tanning) and incidental exposure (WHO, 2016). It is estimated that UV radiation causes $86 \%$ of malignant melanoma cases in the UK (Cancer Research UK, 2016). Thus, skin cancer is to a large degree behaviourally preventable, meaning that developing strategies to reduce UV exposure could be effective in limiting new incidences (Jackson \& Aiken, 2006).

Although the health-related costs of UV exposure and the benefits of sun protection are relatively well known (Miles et al., 2005), interventions that highlight these consequences are not sufficiently effective (Mahler et al., 2006). A possible reason for this is that tanning behaviour is primarily motivated by a desire to improve appearance, and, as such, it is perhaps less responsive to health warnings (McWhirter, 2015). Research on both men and women suggests that focusing on the appearance-related costs of UV exposure is effective in reducing UV exposure (Grogan et al., 2015). A review by Dodd and Forshaw (2010) found that appearance-based interventions were generally successful in improving UV protective behaviours (e.g. sun protection use), but only moderately successful in altering behaviours relating to UV exposure. Another systematic review by McWhirter (2015) found that visual images, e.g. UV photography (i.e. showing participants current level of UV damage to their skin), were successful in promoting sun protection and reducing UV exposure.

The current study is modelled on the Williams et al. (2013) review and meta-analysis, which focused on the efficacy of appearance-based interventions to reduce UV exposure. 


\section{APPEARANCE INTERVENTIONS TO REDUCE UV EXPOSURE}

Williams et al. (2013) found an overall positive impact of appearance-based interventions on reducing UV exposure, and the meta-analysis indicated that UV photo (i.e. demonstrating actual UV damage to a participant's face) and/or photoaging information (i.e. providing participants with information about the ageing effect of the sun), had a significant effect on sun protection intentions and future indoor tanning behaviour. The authors identified a number of problems with the data set, including limited long-term follow-ups, homogeneity of settings, and limited priori power analysis. The current study aims to provide an updated review of the literature, which is considered relevant as the last data search was executed over five years ago. This is particularly important as research into appearance-focused interventions has developed significantly since this time, for instance by including novel techniques such as facial morphing (a type of intervention demonstrating potential future UV damage, by morphing a current image of the person using a specialised software). The current study also includes a larger meta-analysis.

1. Do appearance-based interventions reduce UV exposure immediately after the intervention and/or long-term?

2. What does new research (i.e. the studies not included in the Williams et al., 2013 paper) add to current understanding of the efficacy of appearance-related interventions to reduce UV exposure, and has the quality of research improved?

\section{Method}

\section{Protocol and registration}

A review protocol was not used, however, the review has been reported in accordance with the PRISMA guidelines. See appendix for the PRISMA checklist.

\section{Eligibility criteria}

Eligibility criteria was identical to William's et al. (2013). Studies included an appearancebased intervention, either in isolation (i.e. assessing scores before and after the intervention) 


\section{APPEARANCE INTERVENTIONS TO REDUCE UV EXPOSURE}

or in comparison with another intervention (or control condition); and were required to adopt a pre-test and post-test design, but not necessarily a randomised controlled design (RCTs). Correlational studies were not included. An appearance-based intervention was defined as an intervention that highlighted negative effects of UV exposure on appearance, such as UV photography or photoaging information. Furthermore, studies had to assess the effects of the intervention on sun seeking and/or sun protective behaviours or intentions. Sun seeking behaviours were defined as behaviour that increased UV exposure, and included spending time in the sun or using indoor tanning booths; sun protective behaviours were defined as behaviour intended to decrease UV exposure, such as sun lotion use or protective clothing. Finally, studies were required to administer a post-test measure to assess the effectiveness of the intervention.

\section{Information sources}

The primary source of articles was Web of Knowledge (Science Citation Index Expanded, Social Sciences Citation Index, Arts \& Humanities Citation Index, Conference Proceedings Citation Index - Science and Social Science, Emerging Sources Citation Index); in addition to this seven other electronic databases (CINAHL, ZETOC, PsycARTICLES, PsycINFO, Medline, OVID, Proquest theses) were accessed to search for studies. To ensure the searched databases provided a relevant literature base, it was confirmed that the list of studies included in the William's et al. (2013) paper were found. An ancestry search, i.e. identifying references that cited the identified papers, was also carried out to identify any missing studies.

\section{Search}

The current study used the same search terms as Williams et al's. (2013), to ensure consistency: '(sun*OR UV) AND (appearance OR age spots OR photoaging OR damage OR wrinkles) AND (skin cancer OR melanoma OR health) AND intervention*AND (sunscreen 
APPEARANCE INTERVENTIONS TO REDUCE UV EXPOSURE

OR protect*OR tan* OR expos*OR prevent*OR behav*)', and included studies conducted Jan $1^{\text {st }} 2005$ - May $16^{\text {th }} 2017$. The search was conducted by the first author. 2005 was used as a starting point as research up until this point was sufficiently covered in previous reviews.

\section{Study selection and data collection process}

Eligibility assessment was performed by the first author (see PRISMA flow chart), but agreed upon by all authors. A total of 170 records were identified through database searches, and a total of 532 records were identified through the ancestry search, yielding a total of 702 screened records. Following this, 655 records were excluded based on irrelevancy and duplicity, leaving a total of 47 papers to be examined. In addition, six studies were excluded because the intervention focused on health consequences of UV exposure (Cheng et al., 2011; Dykstra, 2007; Hernandez et al., 2014; Lazovich et al., 2013; Olson et al., 2008 [included in the William's et al. (2013) review]; Thomas et al., 2011), three due to not examining relevant research questions (Cox et al., 2009; Hillhouse et al., 2010; Walsh et al., 2014), and seven for not containing an intervention (Cheetham \& Ogden, 2016; Hillhouse et al., 2016; Noar et al., 2015; Pagoto et al., 2009; Taylor et al., 2016; Welch et al., 2016; Williams et al., 2013b). To identify potential prominent authors in this field were contacted (e.g. authors of the William's et al., 2013 study) and asked whether they had any unpublished material. Additionally, ProQuest theses was searched for unpublished material. An extraction table was designed based on the main elements reported in the Williams et al. (2013) study. Data were extracted by the first author, with $10 \%$ checked blind (i.e. independently extracted by another member of the team and then compared to the data extraction conducted by the first author) by the sixth author during April - May 2017. Due to the high level of agreement $(88 \%)$, the remainder of the data were checked non-blind by the same author, with agreement of $94 . \%$. Any disagreements were resolved by discussion. All papers were further checked and agreed upon by the second author. The final review includes 30 separate articles (33 


\section{APPEARANCE INTERVENTIONS TO REDUCE UV EXPOSURE}

independent studies, as some articles reported more than one study); 18 of these papers (20 individual studies) were not included in the Williams et al. (2013) article. Information extracted from the studies included participant characteristics, study location and settings; intervention characteristics, outcome measures, and which, if any, theoretical constructs were utilised to inform the intervention, and methodological issues.

A formal tool was not utilised to assess methodological bias, but the first author assessed risk of bias in each study by examining the methodology (i.e. study design, proposed analyses, type of intervention, comparison groups etc.), randomization process, quality of the outcome measures (e.g. Cronbach's alpha), and research funding. No studies were deemed to be biased, aside from the Bae et al. (2017) paper, as it was neither controlled nor randomised, and did not compare the intervention with a control condition. However, this risk of bias did not adversely impact the meta-analysis, as it was excluded due to lack of sufficient details for effect-size calculation, and was therefore only commented on in the systematic review. In addition, small study bias and publication bias were assessed utilising Egger's regression (Stern \& Egger, 2000) and trim and fill analyses (Duval \& Tweedie, 2001). This was reviewed and agreed upon by the second and last authors. In sum, the main outcomes of interest included sun seeking behaviours and intentions (i.e. indoor and outdoor tanning), and sun protective behaviours and intentions (i.e. use of protective clothing or sun tan lotion).

\section{Meta-analytical strategy}

The meta-analysis employed a random effects model. All but one of the studies included in the review were also included in the meta-analysis. Bae et al. (2017) was not included as the main author declined a request for additional data to facilitate effect size calculations. Three studies (Mahler et al., 2006; Mahler et al., 2007; Mahler et al., 2013) included separate UV photo and photoaging information components (with the same participants), hence were added as two separate studies under the two relevant interventions. Studies were categorised 


\section{APPEARANCE INTERVENTIONS TO REDUCE UV EXPOSURE}

according to the type of appearance intervention, creating four separate data-sets: interventions with UV photo, photoaging information, UV photo in combination with photoaging information, and interventions that could not be classified as either, for instance facial morphing or group discussions. Due to the heterogeneous nature of the final category, it was not possible to further distinguish between these interventions. The process of categorisation into types of interventions enabled the inclusion of the same participants in separate analyses. In addition, studies described in Gibbons (2005) were originally analysed as one by that paper's authors, resulting in a total of 34 independent studies included in the meta-analysis. For each of these studies, correlation coefficient $r$ was calculated to assess the relationship between the appearance-based intervention and the outcome variable, which was classified as sun protection or UV exposure. Following Cohen's (1992) recommendations, $r$ $=.10$ was taken to represent a 'small' effect size, $r=.30 \mathrm{a}$ 'medium' effect size and $r=.50 \mathrm{a}$ 'large' effect size. Long-term (i.e. any follow-up longer than immediately following the intervention, ranging from one week to six months) effects of the interventions are commented on in the systematic paper review as there was not enough studies with similar levels of follow-ups to include this as a moderator analysis.

Where studies contained two (or more) conditions, the appearance-focused condition was defined as the one with the strongest focus on appearance, and the control condition contained, where possible, active element (e.g. another intervention, as compared to a passive control being waitlist only). Where studies contained more than one appearance-focused intervention, these were compared separately to a control condition, creating separate effect sizes. Where studies lacked relevant statistics, authors were contacted to provide additional information that could facilitate the effect size calculations. All authors except one (Bae et al., 2017) responded with the requested information (Christensen et al., 2014; Cornelis et al., 2014; Gibbons et al., 2005; Hevey et al., 2010; Mahler et al., 2010; Morris et al., 2014; 


\section{APPEARANCE INTERVENTIONS TO REDUCE UV EXPOSURE}

Sontag \& Noar, 2017; Stapleton et al., 2010). These authors were also asked about any unpublished material they might have. As the majority of the studies included a follow-up immediately after the intervention, where possible, this point in time was used to calculate effect sizes to ensure homogeneity of the data. For studies that did not have an immediate follow-up $(N=7)$, or did not report sufficient data for this point, effect sizes were calculated for the nearest available time following the intervention.

The meta-analysis assessed the effectiveness of the intervention on four specific outcome variables: sun protective intentions, sun protective behaviour, UV exposure intentions, and UV exposure. In addition, effectiveness was also assessed as a weighted-mean for multiple outcome variables, henceforth referred to as a combined outcome variable. If multiple outcomes were measured for one of the categories above, e.g., both sun exposure and sun lotion were measured to examine sun protective behaviour, an overall effect size was

calculated as the weighted-mean of these measures. Random effect sizes were computed using SPSS version 22, and the macros developed by Wilson (2005). Effect sizes were weighted by sample, with a $95 \%$ confidence interval, and an estimate of heterogeneity. Publication bias and small study bias were also assessed (Duval \& Tweedie, 2000; Stern \& Egger, 2000)

\section{Results}

\section{Descriptive features of the studies}

\section{Participants and settings}

Across all samples, there were 7348 participants, with sample sizes ranging from 50 to 965 participants. Twelve studies specifically targeted females, whereas four studies targeted males. The remainder had a mixed-gender participant group. Twelve studies based their sample size on power calculations. The majority of the studies included participants aged between 16-35 years. Participants were predominately White. Seven studies targeted a risk 
APPEARANCE INTERVENTIONS TO REDUCE UV EXPOSURE

group such as indoor tanners or highway workers A majority (75.8\%) of the interventions were implemented in a research facility or University setting, with the remainder $(24.2 \%)$ being administered online or in a community setting (e.g. a public beach).

\section{Appearance-based interventions}

The most common type of intervention $(\mathrm{N}=17)$ was UV photography, either in isolation or combined with information about photoaging. Three of the UV photo studies (Mahler et al., 2006; Mahler et al., 2007; Mahler et al., 2013) administered two separate interventions on UV-photo and photoaging. The second most common type of intervention $(\mathrm{N}=7)$ was photoaging information. The remainder of the studies utilised alternative types of interventions, such as discussing and challenging the tanned ideal, manipulating media images or implementing facial morphing. Twenty-one of the studies based their interventions fully or in part on theory. See Table S1 for full details of the theoretical basis and critical points for each of the studies.

\section{Measures employed}

All studies administered post-intervention measures to assess the effect of an appearancebased intervention on UV exposure intentions and/or behaviours. All but one (Bae et al., 2017) of the studies compared this to a control condition (passive control in six of the studies). All of the papers utilised some form of self-report measure to assess intervention efficacy. An alternative method to assess behavioural efficacy of the intervention examines skin colour. It involves the use of a skin reflectance spectrophotometer which, when based on hue lightness and saturation on various skin sites, can indicate level of UV exposure (Mahler et al., 2006). This technique was utilised by four studies.

\section{Descriptive results from systematic review}

Table 1 provides a summary of the overall pattern of findings. Table S2 provides a detailed description of the individual studies, including intervention design and findings. Overall, a 


\section{APPEARANCE INTERVENTIONS TO REDUCE UV EXPOSURE}

majority $(\mathrm{N}=29)$ of the studies reported that an appearance-focused intervention had a positive effect on reducing UV exposure and/or increasing sun protection. Interestingly, four of the studies that reported positive findings only found this effect when examining a particular participant group or combination of conditions; Cornelis et al. (2014) found that an appearance intervention decreased intentions to tan when the argument against tanning was two-sided, but not when it was one-sided; Stapleton et al. (2010) found that their intervention decreased indoor tanning frequency among a sub-group of tanners with previously low knowledge of the health or appearance costs of tanning; and Walsh and Stock (2012) found than UV photo increased sun protection willingness among masculine men. Finally, Morris et al. (2014b) found that UV photo had a positive effect on sun protection intentions only when participants were primed with mortality.

For the studies including a longer (i.e. longer than immediately following the intervention) follow-up, the findings were generally positive. Up until a month after the intervention, participants reduced indoor and outdoor sunbathing frequency and increased use of sun protection (Chait, 2015; Gibbons, 2005a; 2005b). These effects were evident for up to six months, including reduced intentions to tan and increased intentions to use sun protection (Hillhouse et al., 2008; Jackson \& Aiken, 2006).

Three studies did not find an effect of the appearance based intervention on the main measured outcome; Christensen et al. (2014) found that participants in the UV photo condition did not progress in UV-protective stages of change long-term, and the healthoriented intervention was significantly more effective in increasing immediate sun-protective intentions; and Hevey et al. (2010) found no significant difference between a health and appearance-framed message on intentions to use sunscreen and sunbeds. Similarly, Sontag, and Noar (2017) reported no difference between a health and appearance-framed message on UV exposure intentions. 


\section{APPEARANCE INTERVENTIONS TO REDUCE UV EXPOSURE}

Pertaining to the second research question regarding the contribution of the 20 studies published since 2012 (i.e. those not included in William's et al., 2013), there was a similar selection of interventions, apart from the inclusion of two studies utilising facial morphing (Owen et al., 2016; Williams et al., 2013). This technique had positive results on participants' sun protection intentions and behaviour when compared to a health literature intervention. Moreover, three of the five studies specifically targeting a male population were found in this sample. Although most research is still conducted on a female sample, this suggests that research into UV exposure is increasingly considering men's motivation to tan and their barriers to sun protection. The majority of these studies reported modest results, or positive findings confined to a particular combination of conditions (e.g. mortality priming or two-sided arguments). This suggests that appearance-focused interventions to reduce UV exposure may need to consider drawing on other aspects of behaviour change or persuasion theory to enhance efficacy.

\section{Results of meta-analysis}

Table 2 presents the summary of the meta-analyses results (with combined effect sizes), and figure 1 plots effect sizes and Standard Errors. The meta-analysis was carried out on four sub-sets categorised according to the type of intervention utilised; this because some participants took part in more than one intervention, thus is was not possible to analyse the sample as one.

Ten studies (Christensen \& Cooper, 2014; Dwyer, 2014; Heckman et al., 2013; Mahler et al., 2013a, Mahler et al., 2006; Mahler et al., 2007a; Morris et al., 2014a, 2014b; Pagoto et al., 2010; Walsh \& Stock, 2012) examined the effectiveness of UV photo on the combined outcome variable, and on sun protective intentions specifically. For the overall effect of this intervention on all outcomes, the combined effect size was small: $r^{+}=.19 ; k=$ $10, N=1,564,95 \% C I: .084$ to $.296, p<.001$. The effect size on sun protective intentions 


\section{APPEARANCE INTERVENTIONS TO REDUCE UV EXPOSURE}

only was also small $r^{+}=.165 ; k=8, N=1,251,95 \% C I$ : .036 to $.295, p=.012$. Effect sizes were heterogeneous, $\mathrm{Q}(9)=35.38, p<.001$.

Four studies (Mahler et al., 2006b; Mahler et al., 2007b, Mahler et al., 2013b, Tuong \& Armstrong, 2014) examined the effectiveness of photoaging information on sun protective behaviour and intentions combined, and sun protective intentions separately. For the overall effects of photoaging on all of the above outcome variables, the combined effect size was medium $r^{+}=.327 ; k=4, N=836,95 \% C I: .206$ to $.447, p<.001$. On sun protection intentions only the effect size was small $r^{+}=.272 ; k=3, N=813, C I=.203$ to $.341, p=.039$. Effect sizes were heterogeneous, $\mathrm{Q}(9)=7.65, p=.054$, using Higgins et al's (2003) proposed significance level of .10.

Six studies (Gibbons et al., 2005; Mahler et al., 2008; Mahler et al., 2010c; Mahler et al., 2005; Sontag \& Noar, 2017; Stock et al., 2009) examined the effectiveness of UV photography combined with photoaging information on a combination of three outcome variables: sun protective behaviour and intentions, and UV exposure, and sun protective intentions separately. For the effectiveness of this intervention on the above outcome variables, the combined effect size was small, $r^{+}=0.261 ; k=6, N=918, C I=.047$ to $.475, p$ $=.017$. The combined effect size on sun protection intentions only was medium, $r^{+}=.424 ; k$ $=3, N=319, C I=.279-.568, p=.023$. Effect sizes were heterogeneous, $\mathrm{Q}(13)=54.89, p$ $<.001$.

Fourteen studies (Chait et al., 2015; Cooper et al., 2014; Cornelis et al., 2014; Hevey et al., 2010; Hillhouse et al., 2008; Hillhouse et al., 2017; Heckman et al., 2017; Jackson \& Aiken, 2006; Mahler et al., 2010a, 2010b; Stapleton et al., 2010; Stapleton et al., 2015; Williams et al., 2013; Owen et al., 2016) examined the effectiveness of interventions not classed as either of the above on a combination of all of the outcome variables, as well as sun protection intentions, UV exposure and UV exposure intentions separately. For the effects of 
APPEARANCE INTERVENTIONS TO REDUCE UV EXPOSURE

these interventions on the above outcome variables, the combined effect size was small, $r^{+}=$ $.191 ; k=14, N=3895, C I=.117$ to $.265, p<.001$. On UV exposure intentions only, the combined effect size was small, $r^{+}=.235 ; k=7, N=1798, C I=.133$ to $.371, p<.001$. On actual UV exposure, the effect size was small, $\mathrm{r}^{+}=.1542, k=6, \mathrm{~N}=1878, \mathrm{CI}=.007$ to .302 , $\mathrm{p}=.040$. Finally, the effect on sun protection intentions was small but non-significant, $\mathrm{r}^{+}=$ $.223 ; k=5, \mathrm{~N}=773, \mathrm{CI}=-.015$ to $.461, \mathrm{p}=.067$. Effect sizes were heterogeneous, $\mathrm{Q}(6)=$ $26.67, p<.001$.

\section{Summary of risk of bias scores}

As only two unpublished studies were included in the analysis, it was not possible to assess publication bias by directly comparing effect sizes of published and unpublished studies. Thus, a trim and fill analysis was performed (Duval \& Tweedie, 2000) using STATA version 11 (STATA Corp, 2009). Results revealed that there was no bias in interventions utilising UV photo, photoaging information or interventions classed as neither. It did, however, reveal a publication bias in interventions utilising UV photo in combination with photoaging information, filling three studies, rendering the results non-significant, $p=.410$. To ensure the meta-analytical effect sizes were not adversely impacted by underpowered studies from relatively small samples, an Egger's regression was also performed (Stern \& Egger, 2001) using STATA version 11 (STATA Corp, 2009). Results revealed no small-study bias in any of intervention types.

\section{Discussion}

\section{Summary of evidence}

The current study provides a valuable contribution to the existing literature, as it includes 20 individual articles (consisting of 22 independent studies) published between 2012 and 2017 that were not included in Williams et al., (2013), providing an updated examination and 


\section{APPEARANCE INTERVENTIONS TO REDUCE UV EXPOSURE}

analysis of current directions within research on appearance-based interventions.

Furthermore, as the meta-analysis contains more individual studies, it represents a more reliable reflection of the effectiveness of these interventions. Additionally, the current review includes two unpublished papers, a factor that goes some way towards counteracting publication bias.

Appearance-based interventions were generally successful in reducing UV exposure, supporting the findings reported by Williams et al. (2013). The inclusion in the current review of research utilising facial-morphing indicates that this could be an effective intervention for behaviour change. However, three studies did not find an effect of appearance-based intervention when compared to a health-based intervention, which was not identified by William's et al. (2013). One observation made in the current review is that two of these studies used active rather than passive control. This therefore calls for further investigation.

The results of the meta-analyses indicate that appearance based interventions were associated with a small positive effect on intentions and behaviours. The largest effect sizes were associated with UV photography combined with photoaging information. These results may indicate that providing individuals with two sources of information - visual and descriptive - with subjective and objective focus, could be an effective way to influence UVrelated behaviours. The component of photoaging information can also be manipulated according to theory, which may be beneficial, as it could enhance health interventions with theoretical constructs. For instance, Mahler et al. (2005) utilised Theory of Alternative Behaviours (Jacard, 1980) by aiming to alter participants' perceptions of UV exposure and providing an alternative to tanning (sun-less tanning products). Other effective theoretical constructs in this sample included Social Comparison Theory (Festinger, 1994) and Theory of Planned Behaviour (Cialdini et al., 1991). As these interventions appeared to be effective 


\section{APPEARANCE INTERVENTIONS TO REDUCE UV EXPOSURE}

in reducing UV exposure and increasing sun protection among students as well as the general public, it is likely they could be widely implemented. However, due to the issue of publication bias in this sample, it is difficult to draw definitive conclusions. Future research could benefit from investigating this issue further, to determine whether two sources of information could increase the effectiveness of appearance-focused interventions in reducing UV exposure.

There are a number of things to consider when interpreting the results of the metaanalysis. The most common outcome variable was sun protective intentions, which limits the conclusions that can be drawn on other variables. Given the relatively small number of studies, it was not possible to include follow-up length as a mediator in the analysis, it is therefore difficult to determine whether the techniques used would have long term effect on behaviour, as well as immediate effect on intentions. Considerable variability of research methodologies (e.g. control group conditions and inclusion/exclusion of darker skin tones) and reporting style (e.g. inclusion of baseline comparisons and non-significant variables) between the studies makes it difficult to directly compare results between the studies. Furthermore, there was a wide span of effect sizes in the sub-set of the meta-analysis which included any intervention that did not utilise UV photo or photoaging information. This suggests that some of these interventions are more effective than others, and should be further investigated in future research. Lastly, the meta analysis identified a publication bias among studies utilising UV photo in combination with photoaging information. We would therefore encourage researchers and journals alike to consider null results for publication.

\section{Sample limitations and recommendations for future research}

While skin cancer incident rates do not differ significantly between genders (Skin Cancer Foundation, 2016), there was an overwhelming majority of female participants. Given that the current review identified only four studies of male participants, future research would 


\section{APPEARANCE INTERVENTIONS TO REDUCE UV EXPOSURE}

benefit from including men in the study population, particularly as men also value a tanned appearance (Cancer Research UK, 2016; Day et al., 2016). As men may perceive tanning and appearance norms in different terms than women, such as reluctance to engage in practices regarded as feminine (Grogan, 2016), future appearance interventions with men may need to consider the role of masculinity. Moreover, study samples were overwhelmingly young (1635 years); as age increases, the risks of skin damage build-up, so it therefore seems relevant to include an older population in future studies (Cancer Research UK, 2016). Most participants were white; as populations with darker skin are by no means immune to skin cancer, future research would benefit from more ethnically diverse samples (Skin Cancer Foundation, 2016). Finally, some studies included a sample where a large number of participants had experienced skin cancer themselves, or known a family member to do so (Mahler et al., 2013), whereas others did not include this as a variable in the analyses; this is a factor that could skew results, and should be considered in future studies.

The majority of the studies were conducted in the USA, raising concerns about generalisability of findings to other areas; they were also conducted in locations with high level of sun exposure (such as Florida), and it might therefore be difficult to predict whether interventions are effective in countries with fewer days of sun. Qualitative research has indicated that people living in locations with fewer hours of sun (such as the UK) associate UV exposure with leisure time and holidays; this may affect the effectiveness of an intervention to impact motivations to reduce UV exposure among these participants (Persson et al., 2017).

Twelve studies based their sample size on a priori power calculations, with the remaining studies stating a lack of power, or not specifying power calculations. This is problematic, as a potential lack of power in a majority of the examined studies may limit the conclusions that can be drawn from their results, as it can over or under-estimate the effect of 


\section{APPEARANCE INTERVENTIONS TO REDUCE UV EXPOSURE}

the intervention, particularly in combination with publication bias (Charles et al., 2009; Minarik et al., 2016). It is therefore recommended that future research consistently include a priori power calculations, as well as comparing any intervention with an active, rather than a passive, control condition.

\section{Conclusions}

This review and meta-analysis provides a valuable perspective on current research on appearance-based interventions to reduce UV exposure. The findings suggest that appearance-based interventions are associated with small positive effects on reducing sunseeking behaviours and/or increasing sun protective behaviours. These results were generally supported by a meta-analysis. With the previously discussed high levels of skin cancer rates across Western Europe and the US, this would suggest that implementation of these interventions could have scope to prevent skin cancer in a large number of people.

We recommend that practitioners who are looking to increase sun protection intentions administer UV photo in combination with photo-aging information, as this was associated with the largest effect size. These interventions could be administered to men and women alike, over a wide age-span, and they appear to be effective when implemented in a clinical and/or research setting.

A number of methodological issues may limit the conclusions that can be drawn from the results. However, within the current context, this review contributes significantly to the existing body of research into appearance-based interventions to reduce UV exposure, and recommends that future research consistently employ a rigorous methodology (e.g. inclusion of power calculations) and focus on more varied outcomes and a diverse sample population from a wider array of cultures. As motivations for UV exposure might differ in populations living in locations with less opportunities for sun exposure, this review specifically 


\section{APPEARANCE INTERVENTIONS TO REDUCE UV EXPOSURE}

recommends that additional future research on the effectiveness of appearance-focused interventions is conducted in places such as the UK and Northern Europe.

\section{References}

$*$ = paper included in review.

Ajzen, I., \& Madden, T. J. (1986). Prediction of goal-directed behaviour:

Attitudes, intentions, and perceived behavioural control. Journal of Experimental Social Psychology, 22(5), $453-474$

*Bae, Y. C., Bae., E. J., Wang, J. \& Gilchrest, B. (2017) Changes in self-perceptions of photoaging severity and skin cancer risk after objective facial skin quality analysis. Journal of Drugs in Dermatology, 16(5), 435-459.

Beck, A. T. (1967) Depression - Clinical Experimental and Theoretical Aspects, New York: Harper and Row 453-474. doi: http://dx.doi.org/10.1016/0022-1031(86)90045-4.

Cancer Research UK. (2016) Skin Cancer - non Melanoma. Retrieved on October $18^{\text {th }} 2016$ from http://www.cancerresearchuk.org/about-cancer/type/skincancer/.

*Chait, S. R., Thompson, J. K., \& Jacobsen, P. B. (2015). Preliminary development and evaluation of an appearance-based dissonance induction intervention for reducing UV exposure. Body Image, 12, 68-72. doi:10.1016/j.bodyim.2014.09.004

Charles, P., Giraudeau, B., Dechartres, A., Baron, G., \& Ravaud, P. (2009). Reporting of sample size calculation in randomised controlled trials: review. BMJ, 338 . doi:10.1136/bmj.b1732

Cheetham, I., \& Ogden, J. (2016). Enhancing sun safety in young women: The relative impact of format and and temporal framing on beliefs and behaviour. Cogent Psychology, 3(1). doi: 10.1080/23311908.2016.1210069 


\section{APPEARANCE INTERVENTIONS TO REDUCE UV EXPOSURE}

Cheng, S., Guan, X., Cao, M., Liu, Y., \& Zhai, S. (2011). Randomized trial of the impact of a sun safety program on volunteers in outdoor venues. Photodermatology Photoimmunology \& Photomedicine, 27(2), 75-80. doi:10.1111/j.16000781.2011.00567.x, $75-80$

*Christensen, K., Champion, J., \& Wagner, C. (2014). The Effects of Appearance-Based and Health-Based Interventions on Sun Protection Attitudes, Intentions, and Behaviours of College Students. Psycho-Oncology, 23, 317-317.

Cialdini, R. B., Kallgren, C. A., \& Reno, R. R. (1991). A focus theory of normative conduct. Advances in Experimental Social Psychology, 24, 201-234

Cohen, J. (1992). A power primer. Psychological Bulletin, 112, 155-159. doi: 10.1037/00332909.112.1.155

*Cooper, D. P., Goldenberg, J. L., \& Arndt, J. (2014). Perceived efficacy, conscious fear of death and intentions to tan: Not all fear appeals are created equal. British Journal of Health Psychology, 19(1), 1-15. doi:10.1111/bjhp.12019

*Cornelis, E., Cauberghe, V., \& De Pelsmacker, P. (2014). Being healthy or looking good? The effectiveness of health versus appearance-focused arguments in two-sided messages. Journal Of Health Psychology, 19(9), 1132-1142.

doi:10.1177/1359105313485310

Cox, C. R., Cooper, D. P., Vess, M., Arndt, J., Goldenberg, J. L., \& Routledge, C. (2009). Bronze Is Beautiful but Pale Can Be Pretty: The Effects of Appearance Standards and Mortality Salience on Sun-Tanning Outcomes. Health Psychology, 28(6), 746-752. doi:10.1037/n0016388

Coyne, J. C., Thombs, B. D., \& Hagedoorn, M. (2010). 


\section{APPEARANCE INTERVENTIONS TO REDUCE UV EXPOSURE}

Ain't necessarily so: Review and critique of recent meta-analyses of behavioural medicine interventions in health psychology. Health Psychology, 29(2), 107. doi:10.1037/a0017633

Day, A. K., Wilson, C. J., Hutchinson, A. D., \& Roberts, R. M. (2016). Australian young adults' tanning behaviour: The role of ideal skin tone and sociocultural norms. Australian Journal of Psychology, n/a-n/a. doi:10.1111/ajpy.12121

Dodd, L. J., \& Forshaw, M. J. (2010). Assessing the efficacy of appearance-focused interventions to prevent skin cancer: a systematic review of the literature. Health Psychology Review, 4(2), 93-111. doi:10.1080/17437199.2010.485393

*Dwyer, L. A. (2014). Enhancing UV photography among men with choice-promoting information. (3607894 Ph.D.), The George Washington University, Ann Arbor. Retrieved from ProQuest Dissertations \& Theses A\&I database.

Dykstra, J. L. (2007). Avoiding reactance: The utility of ultraviolet photography, persuasion, and parental protectiveness in improving the effectiveness of a UV exposure intervention. (3274866 Ph.D.), Iowa State University, Ann Arbor. Retrieved from ProQuest Dissertations \& Theses A\&I database.

Duval, S. \& Tweedie, R. (2000). Trim and fill: A simple funnel-plot-based method of testing and adjusting for publication bias in meta-analysis. Biometrics, 56(2), 455-463. Festinger, L. (1957). A theory of cognitive dissonance. Evanstone, IL: Row, Peterson

Fishbein, M. Hennessey, M., Yzer, M. \& Douglas, J. (2003). Can we explain why some people do and some people do not act on their intentions? Psychology Health \& Medicine, 8 (1), 3-18. DOI: http://dx.doi.org/10.1080/1354850021000059223

Gibbons, F.X. \& Gerrard, M. (1995). Predicting young adults' health risk behavior. Journal of Personality and Social Psychology, 69, 505-517. DOI: 10.1037//00223514.69.3.505 
APPEARANCE INTERVENTIONS TO REDUCE UV EXPOSURE

Gibbons, F. X., Gerrard, M., Blanton, H., \& Russell, D. W. (1998). Reasoned Action and Social Reaction: Willingness and Intention as Independent Predictors of Health Risk. Journal of Personality and Social Psychology, 74(5), 1164-1180. doi:10.1037/0022-3514.74.5.1164

*Gibbons, F. X., Gerrard, M., Lane, D. J., Mahler, H. I. M., \& Kulik, J. A. (2005). Using UV photography to reduce use of tanning booths: A test of cognitive mediation. Health Psychology, 24(4), 358-363. doi:10.1037/0278-6133.24.4.358

Greenberg, J., Pyszczynski, T., \& Solomon, S. (1986). The Causes and Consequences of a Need for Self-Esteem: A Terror Management Theory. In R. F. Baumeister (Ed.), Public Self and Private Self (pp. 189-212). New York, NY: Springer New York.

Grogan, S. (2016). Body Image. London: Routledge.

Grogan, S., Loosemore, E. (2015). Men's Accounts of Reactions to Two Sources of Information on Negative Effects of UV Exposure: Facial Morphing and a Health Promotion Fact Sheet. International Journal of Men's Health, 14(2).

*Heckman, C., Zhu, F., Manne, S. Kloss, J., Collins, B., Bass, S., Lessin, S. (2013). Process and outcomes of a skin protection intervention for young adults. Journal of Health Psychology, 18(4), 561-573. doi: 10.1177/1359105312449193

*Heckman, C., Handorf, E., Darlow, S., Ritterband, L. \& Manne, S. (2017). An online skin cancer tisk-reduction intervention for young adults: Mechanisms of effect. Health Psychology, 36 (3), 215-225.

Hernandez, C., Wang, S., Abraham, I., Angulo, M. I., Kim, H., Meza, J. R., . . . Uddin, S. (2014). Evaluation of Educational Videos to Increase Skin Cancer Risk Awareness and Sun-Safe Behaviours Among Adult Hispanics. Journal of Cancer Education, 29(3), 563-569. doi:10.1007/s13187-014-0624-z

Hevey, D., Pertl, M., Thomas, K., Maher, L., Craig, A., \& Chuinneagain, S. N. (2010). Body Consciousness Moderates the Effect of Message Framing on Intentions to Use 
APPEARANCE INTERVENTIONS TO REDUCE UV EXPOSURE

Sunscreen. Journal Of Health Psychology, 15(4), 553-559.

doi:10.1177/1359105309355335

Higgins, J., Thompson, S., Deeks, J. \& Altman, D. (2003). Measuring inconsistency in metaanalyses. $B M J, 327(7414), 557-560$.

*Hillhouse, J., Turrisi, R., Stapleton, J., \& Robinson, J. (2008). A Randomized Controlled Trial of an Appearance-focused Intervention to Prevent Skin Cancer. Cancer, 113(11), 3257-3266. doi:10.1002/cncr.23922

Hillhouse, J., Turrisi, R., Stapleton, J., \& Robinson, J. (2010). Effect of Seasonal Affective Disorder and Pathological Tanning Motives on Efficacy of an Appearance-Focused Intervention to Prevent Skin Cancer. Archives Of Dermatology, 146(5), 485-491.

Hillhouse, J., Turrsi, R., Cleveland, M. Scaglione, N., Baker, K. \& Florence, C. (2016). Theory-driven longitudinal study exploring indoor tanning initiation in teens using a a person-centered approach. Annals of Behavioral Medicine, 50(1), 48-57. doi: $10.1007 / \mathrm{s} 12160-015-9731-2$

*Hillhouse, J., Turrsi, R., Scaglione, N., Cleveland, M., Baker K. \& Florence, C. (2017). A web-based intervention to reduce indoor tanning motivations in adolescents: A randomised controlled trial. Prevention Science, 18(2), 131-140. doi: $10.1007 / \mathrm{s} 11121-016-0698-4$

Jaccard, J. (1981). Atttudes and behaviour: Implications of attitudes toward behavioural alternatives. Journal of Experimental Social Psychology, 17(3), 286-307. doi:http://dx.doi.org/10.1016/0022-1031(81)90029-9

Jackson, K. M., \& Aiken, L. S. (2000). A Psychosocial Model of Sun Protection and Sunbathing in Young Women: The Impact of Health Beliefs, Attitudes, Norms, and Self-Efficacy for Sun Protection. Health Psychology, 19(5), 469-478.

doi:10.1037/0278-6133.19.5.469 


\section{APPEARANCE INTERVENTIONS TO REDUCE UV EXPOSURE}

*Jackson, K. M., \& Aiken, L. S. (2006). Evaluation of a multicomponent appearance-based sun-protective intervention for young women: Uncovering the mechanisms of program efficacy. Health Psychology, 25(1), 34-46. doi:10.1037/0278-6133.25.1.34

Kahneman, D., \& Tversky, A. (1979). Prospect theory: An analysis of decisions under risk. Econometrica, 47, 313-327.

Lazovich, D., Choi, K., Rolnick, C., Jackson, J. M., Forster, J., \& Southwell, B. (2013). An Intervention to Decrease Adolescent Indoor Tanning: A Multi-Method Pilot Study. Journal of Adolescent Health, 52(5), S76-S82. doi:10.1016/j.jadohealth.2012.08.009

Löckenhoff, C. E., De Fruyt, F., Terracciano, A., McCrae, R. R., De Bolle, M., Costa, P. T., . . . Yik, M. (2009). Perceptions of Aging across 26 Cultures and their Culture-Level Associates. Psychology and aging, 24(4), 941-954. doi:10.1037/a0016901

Maddux, J.E., \& Rogers, R. W. (1983). Protection motivation theory and self-efficacy: A revised theory of fear appeals and attitude change. Journal of Experimental Social Psychology, 19, 469-479.

*Mahler, H. I. M., Beckerley, S. E., \& Vogel, M. T. (2010). Effects of Media Images on Attitudes Toward Tanning. Basic and Applied Social Psychology, 32(2), 118-127. doi:10.1080/01973531003738296

*Mahler, H. I. M., Kulik, J. A., Butler, H. A., Gerrard, M., \& Gibbons, F. X. (2008). Social norms information enhances the efficacy of an appearance-based sun protection intervention. Social Science \& Medicine, 67(2), 321-329. doi:10.1016/j.socscimed.2008.03.037

*Mahler, H. I. M., Kulik, J. A., Gerrard, M., \& Gibbons, F. X. (2006). Efffects of two appearance-based interventions on the sun protection behaviours of southern California beach patrons. Basic and Applied Social Psychology, 28(3), 263-272. doi:10.1207/s15324834basp2803_5 
APPEARANCE INTERVENTIONS TO REDUCE UV EXPOSURE

*Mahler, H. I. M., Kulik, J. A., Gerrard, M., \& Gibbons, F. X. (2007). Long-term effects of appearance-based interventions on sun protection behaviours. Health Psychology, 26(3), 350-360. doi:10.1037/0278-6133.26.3.350

*Mahler, H. I. M., Kulik, J. A., Gerrard, M., \& Gibbons, F. X. (2010). Effects of upward and downward social comparison information on the efficacy of an appearance-based sun protection intervention: a randomized, controlled experiment. Journal Of Behavioural Medicine, 33(6), 496-507. doi:10.1007/s10865-010-9279-3

*Mahler, H. I. M., Kulik, J. A., Gerrard, M., \& Gibbons, F. X. (2013). Effects of photoaging information and UV photo on sun protection intentions and behaviours: A crossregional comparison. Psychology \& Health, 28(9), 1009-1031. doi:10.1080/08870446.2013.777966

Martin, J. M., Ghaferi, J. M., Cummins, D. L., Mamelak, A. J., Schmults, C. D., Parikh, M., Liégeois, N. J. (2009). CHANGES in SKIN TANNING ATTITUDES Fashion Articles and Advertisements in the Early 20th Century. American Journal of Public Health, 99(12), 2140-2146. doi:10.2105/AJPH.2008.144352

McWhirter, J. E., \& Hoffman-Goetz, L. (2015). Systematic review of population-based studies on the impact of images on UV attitudes and behaviours. Health Promotion International, 30(2), 397-410. doi:10.1093/heapro/dat031

Miles, A., Waller, J., Hiom, S., \& Swanston, D. (2005). SunSmart? Skin cancer knowledge and preventive behaviour in a British population representative sample. Health Educ Res, 20(5), 579-585. doi:10.1093/her/cyh010

Minarik, T., Berger, B., Althaus, L., Bader, V., Biebl, B., Brotzeller, F., . . Sauseng, P. (2016). The Importance of Sample Size for Reproducibility of tDCS Effects. Frontiers in Human Neuroscience, 10, 5. doi:10.3399/fnhum.2016.00453 
*Morris, K. L., Cooper, D. P., Goldenberg, J. L., Arndt, J., \& Gibbons, F. X. (2014). Improving the efficacy of appearance-based sun exposure interventions with the terror management health model. Psychology \& Health, 29(11), 1245-1264. doi:10.1080/08870446.2014.922184

Noar, S. M., Myrick, J. G., Zeitany, A., Kelley, D., Morales-Pico, B., \& Thomas, N. E. (2015). Testing a Social Cognitive Theory-Based Model of Indoor Tanning: Implications for Skin Cancer Prevention Messages. Health Communication, 30(2), 164-174. doi:10.1080/10410236.2014.974125

Olson, A. L., Gaffney, C. A., Starr, P., \& Dietrich, A. J. (2008). The impact of an appearance-based educational intervention on adolescent intention to use sunscreen. Health Education Research, 23(5), 763-769 767p. doi:her/cym005

*Owen, A. (2016). Effects of an appearance-focussed versus a health-focussed intervention on men's attitudes towards UV exposure. International Journal of Men's Health.

*Pagoto, S. L., Schneider, K. L., Oleski, J., Bodenlos, J. S., \& Ma, Y. (2010). The Sunless Study A Beach Randomized Trial of a Skin Cancer Prevention Intervention Promoting Sunless Tanning. Archives Of Dermatology, 146(9), 979-984.

Pagoto, S. L., Schneider, K. L., Oleski, J., Bodenlos, J. S., Merriam, P., \& Ma, Y. (2009). Design and methods for a cluster randomized trial of the Sunless Study: A skin cancer prevention intervention promoting sunless tanning among beach visitors. Bmc Public Health, 9. doi:10.1186/1471-2458-9-50

Persson, S., Benn, Y., Dhingra, K. \& Grogan, S. (2017). "It's a bit of an eye opener": A qualtitative study of women's attitudes towards tanning, sun protection and a facial morphing intervention. Psychology \& Health, 1-17, doi: http://dx.doi.org/10.1080/08870446.2017.1357815

Petty, R. E., \& Cacioppo, J. T. (1984). Source Factors and the Elaboration Likelihood Model of 
APPEARANCE INTERVENTIONS TO REDUCE UV EXPOSURE

Persuasion. Advances in Consumer Research, 11, 668.

PRISMA (2011). Transparent Reporting of Systematic Reviews and Meta-Analyses.

Retrieved on October 18, 2016 from http://www.prisma-statement.org/index.htm

Prochaska, J. O., \& DiClemente, C. C. (1983). Stages and processes of self-change of smoking:

Toward an integrative model of change. Journal of Consulting \& Clinical Psychology, 51(3), 390-395.

Rosenstock, I. M. (1974). Historical Origins of the Health Belief Model. Health Education \& Behaviour, 2(4), 328-335. doi:10.1177/109019817400200403.

Skin Cancer Foundation. (2016). Skin Cancer Facts \& Statistics. Retrieved October, $18^{\text {th }}$ 2016 from http://www.skincancer.org/skin-cancer-information/skin-cancerfacts\#ethnicity

*Sontag, J. \& Noar, S. (2017). Assesing the potential effectiveness of pictoral messages to deter young women from indoor tanning: an experimental study. Journal of Health Communication, 22(4), 294-303. doi: 10.1080/10810730.2017.1281361

*Stapleton, J., Turrisi, R., Hillhouse, J., Robinson, J. K., \& Abar, B. (2010). A comparison of the efficacy of an appearance-focused skin cancer intervention within indoor tanner subgroups identified by latent profile analysis. Journal Of Behavioural Medicine, 33(3), 181-190. doi:10.1007/s10865-009-9246-Z

*Stapleton, J. L., Manne, S. L., Darabos, K., Greene, K., Ray, A. E., Turner, A. L., \& Coups, E. J. (2015). Randomized Controlled Trial of a Web-Based Indoor Tanning Intervention: Acceptability and Preliminary Outcomes. Health Psychology, 34, 1278-1285. doi:10.1037/hea0000254

StataCorp. (2009). Stata statistical software (11th edition). Dallas, TX: College Station. *Stock, M. L., Gerrard, M., Gibbons, F. X., Dykstra, J. L., Mahler, H. I. M., Walsh, L. A., \& Kulik, J. A. (2009). Sun Protection Intervention for Highway Workers: Long-Term 
APPEARANCE INTERVENTIONS TO REDUCE UV EXPOSURE

Efficacy of UV Photography and Skin Cancer Information on Men's Protective Cognitions and Behaviour. Annals of Behavioural Medicine, 38(3), 225-236. doi:10.1007/s12160-009-9151-2

Taylor, M., Westbrook, D. \& Chang, P. (2016). Using UV photoaged photography to better understand Western Australian teenagers' attitudes towards adopting sun-protective behaviours. International Journal of Adolescent Medicine and Health, 28(1), 45-53. doi: 10.1515/ijamh-2014-0071

Thomas, K., Hevey, D., Pertl, M., Ni Chuinneagain, S., Craig, A., \& Maher, L. (2011). Appearance matters: The frame and focus of health messages influences beliefs about skin cancer. British Journal of Health Psychology, 16, 418-429.

*Tuong, W., \& Armstrong, A. W. (2014). Effect of appearance-based education compared with health-based education on sunscreen use and knowledge: A randomized controlled trial. Journal of the American Academy of Dermatology, 70(4).

*Walsh, L. A., \& Stock, M. L. (2012). UV photography, masculinity, and college men's sun protection cognitions. Journal Of Behavioural Medicine, 35(4), 431-442.

Walsh, L. A., Stock, M. L., Peterson, L. M., \& Gerrard, M. (2014). Women's sun protection cognitions in response to UV photography: the role of age, cognition, and affect. Journal Of Behavioural Medicine, 37(3), 553-563.

Welch, M., Chang, P. \& Taylor, M. (2016). Photoaging photopgraphy: Mothers' attutides toward adopting skin-proective measuers pre-and post -viewing photoaged images of their and their child's facial sun damage. Sage Open, 6(4). doi: $10.1177 / 2158244016672906$.

Williams, A. L., Grogan, S., Buckley, E., \& Clark-Carter, D. (2013). Men's experiences of an appearance-focussed facial-ageing sun protection intervention: A qualitative study. Body Image, 10(2), 263-266. doi:10.1016/j.bodyim.2013.01.003 
Williams, A. L., Grogan, S., Clark-Carter, D., \& Buckley, E. (2013). Appearance-based interventions to reduce ultraviolet exposure and/or increase sun protection intentions and behaviours: a systematic review and meta-analyses. British journal of health psychology, 18(1), 182.

*Williams, A. L., Grogan, S., Clark-Carter, D., \& Buckley, E. (2013). Impact of a facialageing intervention versus a health literature intervention on women's sun protection attitudes and behavioural intentions. Psychology \& Health, 28(9), 993-1008.

Wilson, D. B. (2005). SPSS macros for meta-analytic data. Retrieved May 15, 2016, from http://mason.gmu.edu/ dwilsonb/ma.html

World Health Organization (WHO). (2016). Skin Cancers. Retrieved October 18, 2016, from http://www.who.int/uv/faq/skincancer/en/index1.html.

Table 1. General summary of results

\begin{tabular}{|c|c|c|c|c|c|c|}
\hline Sample & Settings & Interventions & $\begin{array}{l}\text { Outcomes } \\
\text { measured }\end{array}$ & Follow-up & Findings & $\begin{array}{l}\text { Theoretical } \\
\text { basis }\end{array}$ \\
\hline $\begin{array}{l}\mathrm{N}=7348 \\
(\mathrm{M}= \\
222.67, \\
\text { Median }= \\
148, \mathrm{SD}= \\
189.96)\end{array}$ & $\begin{array}{l}75.8 \% \\
\text { Research } \\
\text { facility or } \\
\text { University }\end{array}$ & $\begin{array}{l}17=\mathrm{UV} \\
\text { photo (with or } \\
\text { without } \\
\text { photoaging } \\
\text { information) }\end{array}$ & $\begin{array}{l}12=\text { Sun } \\
\text { protective } \\
\text { intentions } \\
\text { and } \\
\text { behaviours }\end{array}$ & $\begin{array}{l}12= \\
\text { Immediately } \\
\text { only }\end{array}$ & $\begin{array}{l}\text { Positive } \\
\text { findings }=29 \\
\text { studies }\end{array}$ & $\begin{array}{l}27= \\
\text { theoretical } \\
\text { basis }\end{array}$ \\
\hline $\begin{array}{l}72.9 \% \\
\text { women }\end{array}$ & $\begin{array}{l}15.2 \% \\
\text { Online }\end{array}$ & $\begin{array}{l}7= \\
\text { photoaging } \\
\text { information }\end{array}$ & $\begin{array}{l}10=\mathrm{UV} \\
\text { exposure } \\
\text { and } \\
\text { intentions }\end{array}$ & $\begin{array}{l}21= \\
\text { between } 1 \\
\text { week and } 12 \\
\text { months }\end{array}$ & $\begin{array}{l}\text { Positive } \\
\text { findings } \\
\text { confined to } \\
\text { specific } \\
\text { participant } \\
\text { group/condition } \\
=4\end{array}$ & $\begin{array}{l}6=\text { no } \\
\text { theoretical } \\
\text { basis }\end{array}$ \\
\hline $\begin{array}{l}12-75 \\
\text { years age } \\
\text { range }\end{array}$ & $9 \%$ Other & $\begin{array}{l}9=\text { neither } \\
\text { UV photo or } \\
\text { photoaging } \\
\text { info }\end{array}$ & $\begin{array}{l}11= \\
\text { combination } \\
\text { of both }\end{array}$ & & $\begin{array}{l}\text { No difference }= \\
4\end{array}$ & \\
\hline $\begin{array}{l}12= \\
\text { utilised } \\
\text { power } \\
\text { calculations }\end{array}$ & & & & & & \\
\hline
\end{tabular}




\section{APPEARANCE INTERVENTIONS TO REDUCE UV EXPOSURE}

\section{Table 2. Meta-analyses results}

Relationship between UV photo interventions and outcome variables

\begin{tabular}{lccccc}
\hline Outcome variable & $\mathrm{r}^{+}$ & $p$ & $95 \%$ confidence interval & $k$ (studies) & $N$ (participants) \\
\hline $\begin{array}{l}\text { Combined } \\
\text { outcome } \\
\text { variable }\end{array}$ & .19 & $<.001$ & $084-.296$ & 10 & 1564 \\
SPI $^{1}$ & .165 & .012 & $.036-.295$ & 8 & 1251 \\
\end{tabular}

Relationship between photoaging interventions and outcome variables

\begin{tabular}{lccccc}
\hline Outcome variable & $\mathrm{r}^{+}$ & $p$ & $95 \%$ confidence interval & $k$ (studies) & $N$ (participants) \\
\hline $\begin{array}{l}\text { Combined } \\
\text { outcome } \\
\text { variable }\end{array}$ & .327 & $<.001$ & $.206-.447$ & 4 & 863 \\
SPI $^{1}$ & & & & & 840 \\
\end{tabular}

Relationship between UV photo combined with photoaging information and outcome variables

\begin{tabular}{llcccc}
\hline Outcome variable & $\mathrm{r}^{+}$ & $p$ & $95 \%$ confidence interval & $k$ (studies) & $N$ (participants) \\
\hline $\begin{array}{l}\text { Combined } \\
\text { outcome } \\
\text { variable }\end{array}$ & .261 & $<.017$ & $.047-.475$ & 6 & 918 \\
SPI & .424 & .023 & $.279-.568$ & 3 & 319 \\
\hline
\end{tabular}

Relationship between other interventions and outcome variables

\begin{tabular}{|c|c|c|c|c|c|}
\hline Outcome variable & $\mathrm{r}^{+}$ & $p$ & $95 \%$ confidence interval & $k$ (studies) & $N$ (participants) \\
\hline $\begin{array}{l}\text { Combined } \\
\text { outcome } \\
\text { variable }\end{array}$ & .191 & $<.001$ & $.117-.265$ & 14 & 3895 \\
\hline SPI & .223 & .067 & $-.015-.461$ & 5 & 836 \\
\hline UV exposure & .154 & .040 & $.007-.302$ & 6 & 1878 \\
\hline UVEI $^{2}$ & .235 & $<.001$ & $.133-.371$ & 7 & 1798 \\
\hline
\end{tabular}

Figure 1. Figures demonstrating of effect sizes and SE

Vertical line demonstrating the combined effect size $\left(\mathrm{r}^{+}\right)$ 


\section{APPEARANCE INTERVENTIONS TO REDUCE UV EXPOSURE}
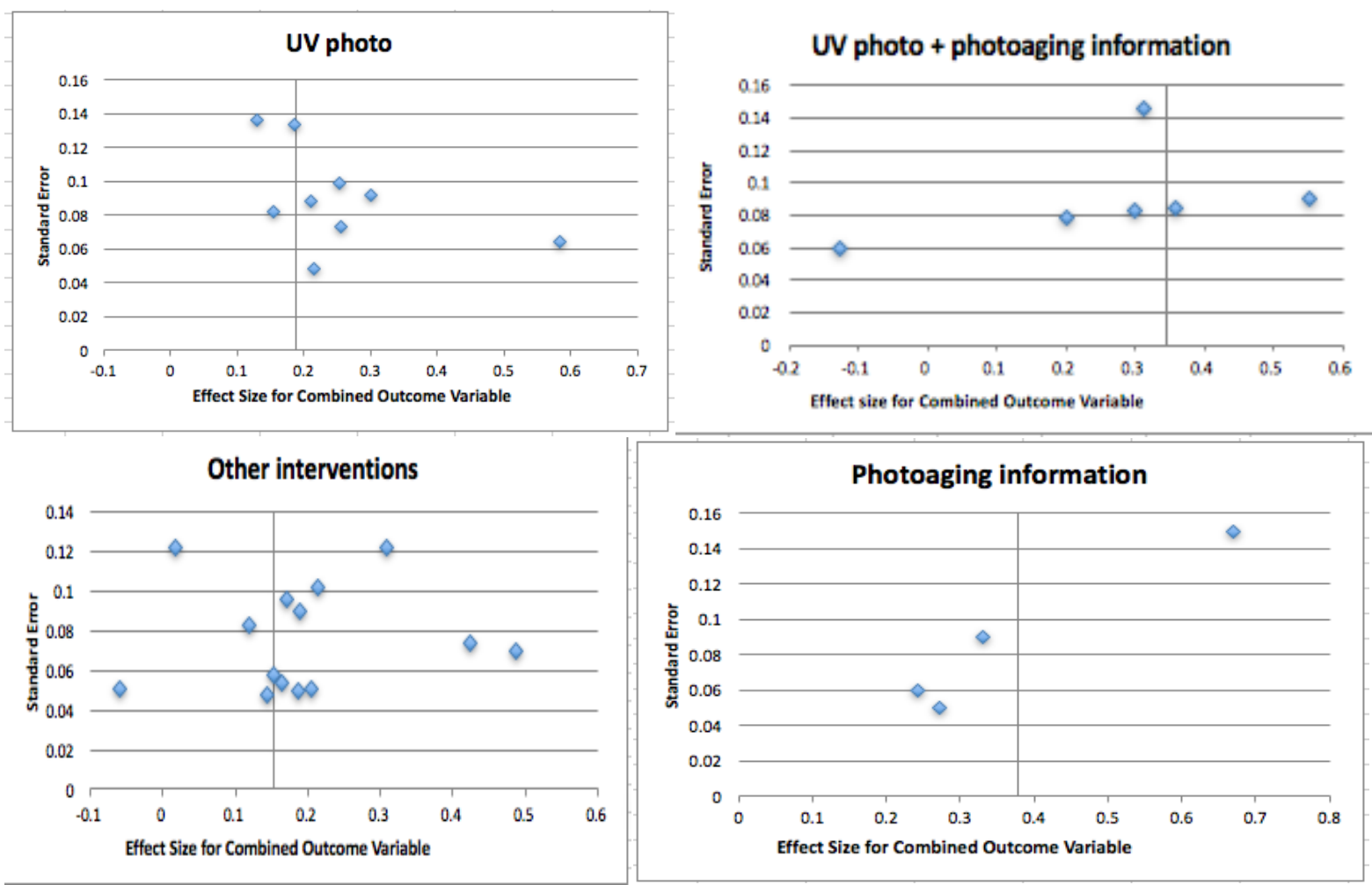

Appendix: PRISMA (2009) flow diagram
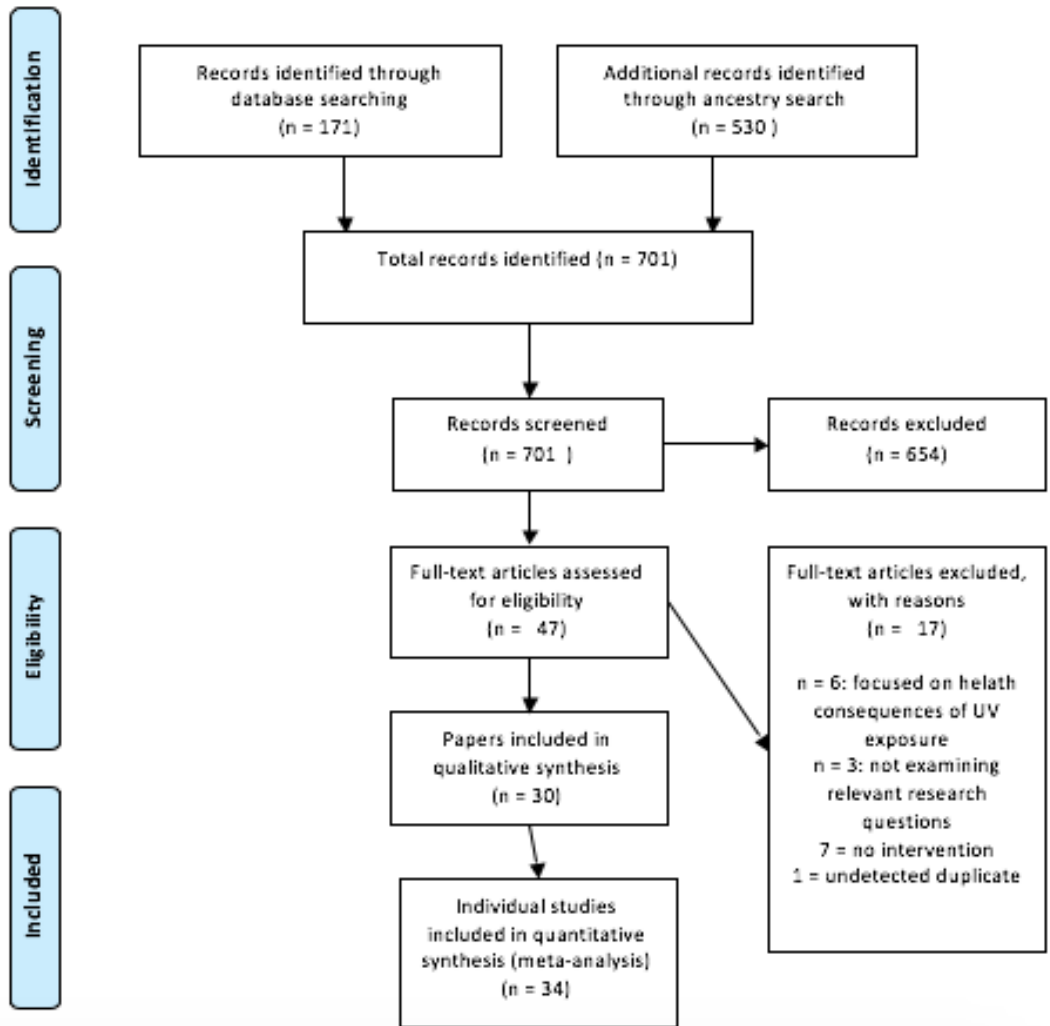